\title{
36
}

\section{On Radiative Transfer Near the Plasma Frequency at Strong Coupling}

\author{
YU. K. KURILENKOV \\ Institute for High Temperatures, Russian Academy of Science, Moscow 127412 \\ H.M. VAN HORN \\ Department of Physics and Astronomy and C. E. Kenneth Mees Observatory, \\ University of Rochester, Rochester, NY 14627-0011, U.S.A.
}

\begin{abstract}
The effects of strong coupling on the frequency-averaged optical characteristics of plasmas, such as the Rosseland mean-free-path, are considered. The general expression for the Rosseland mean opacity has been analyzed in terms of the transverse dielectric function of a dense plasma and the frequency-dependent effective collision frequency. The corresponding values of the absorption coefficient and the refractive index for a dense plasma are presented at $\omega \leq \omega_{p}$ in obvious forms.
\end{abstract}

\subsection{Introduction}

We are concerned with rather "cold," classical, strongly coupled plasmas (SCPs), where the energy $\hbar \omega_{p}$ of a plasmon is comparable to or higher than the thermal energy of the electrons. The electron plasma frequency is $\omega_{p}=\left(4 \pi n_{e} \epsilon^{2} / m_{e}\right)^{1 / 2}$. The plasma under consideration is specified by two parameters: (1) the ion coupling parameter

$$
\Gamma=(Z e)^{2} \beta / a \geq 1,
$$

where $\beta=1 / k_{B} T$ is the inverse temperature, $Z$ is the ionic charge, and $a=\left(3 / 4 \pi n_{i}\right)^{1 / 3}$ is the ion sphere radius, and (2) the electron degeneracy parameter

$$
\theta=1 / \beta E_{F}=2(4 / 9 \pi)^{2 / 3} Z^{5 / 3} r_{s} / \Gamma>1,
$$

where $E_{F}=\left(3 \pi^{2} n_{\epsilon}\right)^{2 / 9} \hbar^{2} / 2 m_{\epsilon}$ is the Fermi energy of electrons at the temperature $T=0$, and $r_{s}=\left(m_{e} c^{2} / \hbar^{2}\right)\left(3 / 4 \pi n_{e}\right)^{1 / 3}$ is the electron density parameter. Under these plasma conditions, nonideality effects in the optical properties are expected to be important. On the other hand, crystallization - which occurs for $\Gamma \geq 180$ - does not yet take place in this modest. 
$\Gamma$ range. We are thus dealing with an intermediate state between ideal and solid-density plasmas. Such plasmas occur frequently both in postmain-sequence phases of stelar evolution (stellar interiors, core-envelope boundaries, and surface areas) and in laboratory experiments with high local concentrations of energy (see Van Horn and Ichimaru 1993).

SCP effects manifest themselves differently in the total absorption coefficient for different frequency domains. We restrict ourselves here mainly to the important, but partial, case when the frequency is $\omega \leq \omega_{p}$. This frequency range is critical for radiative transfer, especially under conditions where $\hbar \omega_{p} \sim k T$. The collective modes are important in spite of the collision-dominated character of the SCP, so we attempt to consider the Rosseland mean absorption in terms of the collision frequency and the dielectric function.

\subsection{Dielectric Function and Dynamic Collision Frequency}

In fact, all the information on the optical properties of a plasma is included in the transverse dielectric function $\varepsilon_{t r}(\omega)$. In turn, the dielectric function depends upon the dynamic electron-ion collision frequency $\nu(\omega)$ :

$$
\varepsilon_{t r}(\omega)=1-\frac{\omega_{p}^{2}}{\omega[\omega+i \nu(\omega)]},
$$

for $\nu(\omega)<\omega$. Explicit theoretical results for $\nu(\omega)$ are well-known in two limiting cases: in classical dilute plasmas $(\Gamma<<1$ and $\theta>>1)$ and in quantum cases $(\theta<<1)$, when the electron-ion interaction is weak enough. The Born approximation, which is acceptable for $\omega \leq \omega_{p e}$ (Ichimaru, Iyetomi, and Tanaka 1987; Berkovsky, Kurilenkov, and Milchberg 1992) gives the following expression for the dynamic collision frequency:

$$
\nu_{\mathrm{Born}}(\omega)=-\frac{1}{3 m_{\epsilon} \omega n_{\varepsilon}} \int \frac{d^{3} q}{(2 \pi)^{3}} q^{2} S_{i i}(q) v_{e i}^{2}(q) \frac{\operatorname{Im} \Pi_{\epsilon \epsilon}(q, \omega)}{\left|\varepsilon_{\varepsilon}(q, \omega)\right|^{2}} .
$$

Here $S_{i i}(q)$ is the ion structure factor, $\Pi_{e \epsilon}(q, \omega)$ is the electron polarization function, and $\varepsilon_{\varepsilon}(q, \omega)=1-v_{\epsilon \varepsilon}(q) \Pi_{\epsilon \varepsilon}(q, \omega)$ is the longitudinal part of the dielectric function of the electron subsystem. Strong coupling effects appear in eq. (4) through the static structure factors $S_{i i}(q)$ and the local field correction factor $G_{\epsilon}(q, \omega)$ in $\varepsilon(q, \omega)$. We used the hypernetted chain (HNC) equations for electronically screened ions to calculate $S_{i i}(q)$, and we evaluated $G_{\varepsilon}(q, \omega) \approx G_{\varepsilon}(q)$ from the HNC equations for a one-component electron system (Berkovsky et al. 1992). The role of the dynamic local 


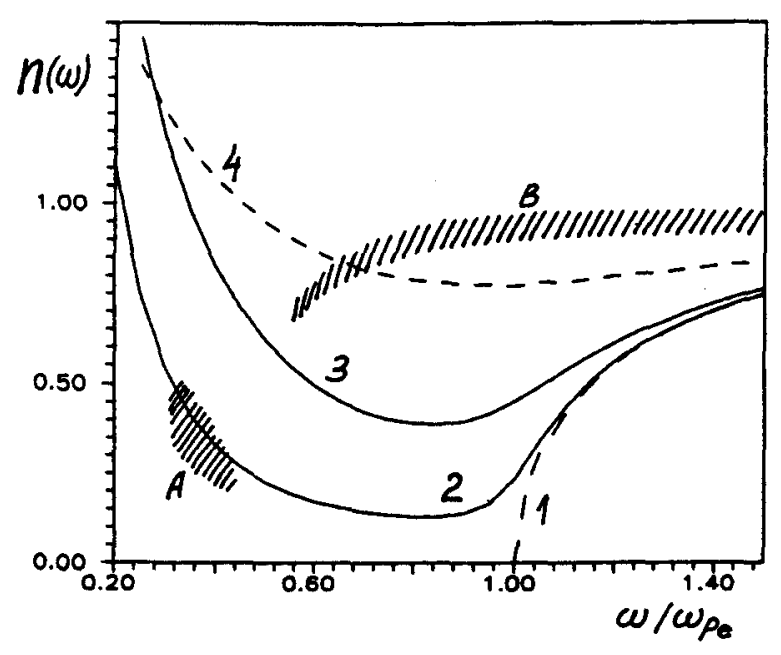

Fig. 36.1 The SCP refractive index $n(\omega)$. Curve 1 gives the results for an ideal plasma; curve 2 for $\Gamma=0.5, \theta \leq 1$; curve 3 for $\Gamma=0.8, \theta \approx 4$; and curve 4 for the case $\nu \equiv \omega_{p}$. The cross-hatched areas give estimates based on the MD results from Sjogren, Hansen, and Pollock $(1981 ; \Gamma=0.5, \theta \approx 1$ : area $A$ ) and from Valuev $(1981 ; \Gamma \approx 1, \theta>>1$ : area $B$ ).

field correction and of non-Born efects are discussed elsewhere (Ichirnaru, Iyetomi, and Tanaka 1987; Berkovsky and Kurilenkov 1993).

\subsection{Refractive Index and Absorption Coefficient at $\omega \leq \omega_{p}$}

From the roots $k(\omega)$ of the dispersion relation $k^{2}(\omega)=(\omega / c)^{2} \varepsilon_{t r}(\omega, k)$, the optical properties of any medium are easily defined: the refractive index $n(\omega) \equiv(c / \omega) R \epsilon k(\omega)$, the absorption coefficient $\kappa(\omega) \equiv I m k(\omega)$, the skindepth for electromagnetic wave penetration into a plasma $L \equiv 1 / \operatorname{Im} k(\omega)$ (see Berkovsky, Kurilenkov, and Milchberg 1992), etc. We suppose that $\omega>>v_{T e}$, and we neglect the $k$-dependence of the transverse dielectric function $\varepsilon_{t r}$ in the discussion below (long-wavelength approximation).

The refractive index and absorption coefficient are essential for calculations of the SCP Rosseland mean-free-path. Some results for $n(\omega)$ and $\kappa_{f f}(\omega)$ are thus presented in Figs. 1 and 2, respectively, corresponding to different SCP parameters. Some estimates of $n(\omega)$ and $\kappa_{f f}(\omega)$ from available molecular dynamics (MD) data for the high-frequency conductivity are also presented. The SCP collective modes at different $\Gamma$ and $\theta$ [in particular, the values of $\nu\left(\omega / \omega_{p}\right)$ ] affect the functions $n(\omega)$ and $\kappa_{f f}(\omega)$, including anomalous absorption and refraction (curves 4 in Figs. 1 and 2 ). 


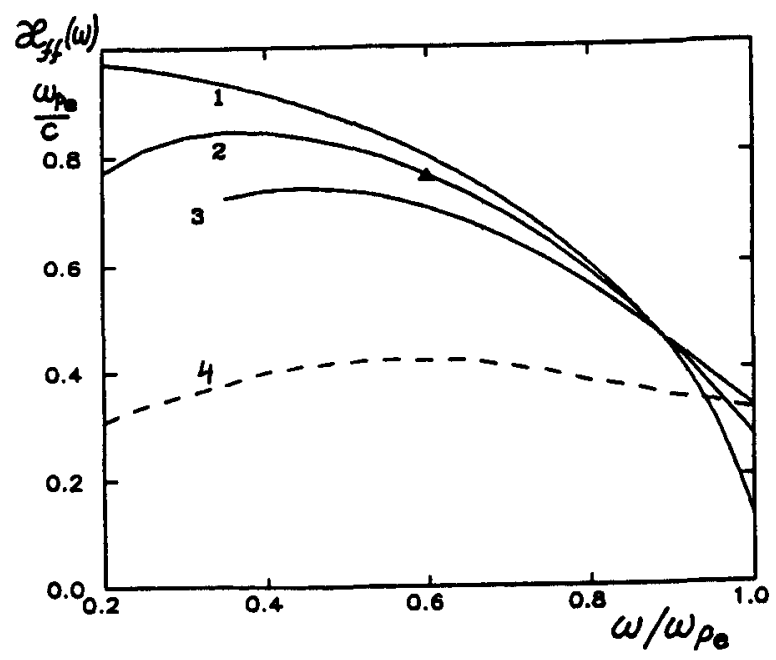

Fig. 36.2 The SCP free-free absorption coefficient $\kappa_{f f}(\omega)$. Curve 1 gives the results for $\Gamma=0.1, \theta \approx 2$; curve 2 for $\Gamma=0.5, \theta \approx 4$; curve 3 for $\Gamma=0.8, \theta \approx 4$; and curve 4 for the case $\nu \equiv \omega_{p}$. The triangle gives an estimate based on the MD results of Furukawa et al. (1990).

\subsection{Rosseland Mean-Free-Path in SCP}

The data presented above can be used to calculate frequency-integrated optical characteristics such as the Rosseland mean-free-path $\ell_{R}$. The SCP effects manifest themselves in different ways in the total absorption coefficient in different frequency domains (Berkovsky et al. 1993). Let us discuss some qualitative features of $\ell_{R}$.

The following expression for the Rosseland mean-free=path, expressed in terms of the transverse dielectric function (i.e. in terms of $n(\omega)$ and $\kappa_{f f}(\omega)$ of dense plasinas, is a new generalization of earlier results (Bekefi 1966; Van Horn 1992):

$$
\ell_{R}=\frac{c \int_{0}^{\infty}\left\{[R \epsilon \sqrt{\varepsilon(\omega)}]^{2} / \omega I m \sqrt{\varepsilon(\omega)}\right\}\left(\partial B_{\omega} / \partial T\right) d \omega}{\int_{0}^{\infty}\left(\partial B_{\omega} / \partial T\right) d \omega},
$$

where $B_{\omega}(T)$ is the usual Planck function. Correspondingly, dense plasma effects in the opacity depend upon the combination of plasma parameters $\Gamma, \theta$, and $\hbar \omega_{p}$ (i.e., upon the charge densities and temperature, as usual). If the peak of the Rosseland factor in eq. (5) corresponds to $\omega \leq \omega_{p}$, then the strong absorption due to collective effects can produce a decrease in $\ell_{R}$ (or increasing opacity) in spite of the collision-dominated character of the plasma. Note that this effectively corresponds to a cut-off of the integration in the numerator of eq. (5) at frequencies less than $\sim \omega_{p}$ (Van Horn 1992). However, when the Planck peak corresponds to $\omega>>\omega_{p}$, the role of 
the collective modes discussed above disappears, and the role of bound-free absorption is reduced significantly (and the free-free absorption turns out to be even less than the Kramers values: Berkovsky et al. 1993). Under these conditions, the Rosseland mean-free-path $\ell_{R}$ may increase in comparison with the ideal value (reduced opacity). Note that corresponding features of radiative transfer, in particular, near the core-envelope boundaries of white dwarfs, probably have important consequences for their cooling (Van Horn 1991).

\section{References}

Bekefi, G. 1966, Radiation Processes in Plasmas (John Wiley \&. Sons, Inc.: New York).

Berkovsky, M. A., and Kurilenkov, Yu. K. 1993, Physica, to be published.

Berkovsky, M. A., Kurilenkov, Yu. K., Kelleher, D., and Skowronek, M. 1993, J. Phys. B: At. Mol: Opt. Phys., 26, 2475.

Berkovsky, M. A., Kurilenkov, Yu. K., Kobzev, G. A., Milchberg, H. M., and Skowronek, M. 1993, in Strongly Coupled Plasma Physics, eds. Van Horn, H. M., and Ichimaru, S. (University of Rochester Press: Rochester, NY), p. 263.

Berkovsky, M. A., Kurilenkov, Yu. K., and Milchberg, H. M. 1992, Phys. Fluids, B4, 2423.

Furukawa, H., Nichihara, K., Kawaguchi, M., Sakagami, H., Hiramatsu, T., and Yasui, H. 1990, in Strongly Coupled Plasma Physics, ed. S. Ichimaru (Elsevier Sci. Publ.: Tokyo), p. 613.

Ichimaru, S., Iyetomi, H., and Tanaka, S. 1987, Phys. Rep., 149, 91.

Sjogren, Hansen, J. P., and Pollock, E. L. 1981, Phys. Rev., A24, 1544.

Valuev, A. A. 1981, High Temp., 19, 200.

Van Horn, H. M. 1991, Science, 252, 384.

Van Horn, H. M. 1992, Bull. A. A. S., 24, 824.

Van Horn, H. M., and Ichimaru, S., eds. 1993, Strongly Coupled Plasma Physics (University of Rochester Press: Rochester, NY). 